\title{
Selective loss of visual and verbal information in STM by means of visual and verbal interpolated tasks*
}

\author{
KEN den HEYER $†$ and BRUCE BARRETT \\ St. Francis Xavier University, Antigonish, N.S., Canada
}

Using incompletely filled grids, Ss retained simultaneously both the position and identity of items for 10-sec periods which were filled with one of three different kinds of interpolated activity. Interpolated activity consisted of (1) no activity, (2) a verbal task involving addition of orally presented digits, or (3) a visual task involving discrimination between dot patterns. Percentage loss data demonstrated that the visual and verbal interpolated conditions were selective in affecting type of information loss, thus suggesting that position and identity information are stored, or at least lost, independently. The fact that the selective effect of information lost as a result of the visual and verbal interpolated task was much greater for position information than for identity information suggests a two-modality STM code. Presumably, position information is mediated by a structural or visual code and identity information is mediated by the same structural code as well as an acoustic code.

Several studies and reviews have indicated that verbal materials such as letters, digits, and words are stored in some acoustic or verbal form in short-term memory (STM), even if such stimulus materials are presented visually (Baddeley, 1964; Conrad, 1964; Conrad \& Hull, 1964; Dale \& Gregory, 1966; Sperling, 1963, 1967). For instance, Conrad (1964) found that the substitution errors made in recalling visually presented letters correlated highly with errors made in identifying letters spoken in a noisy background. This suggests that letters encoded from visual storage, or iconic storage (Neisser, 1967), are stored in some auditory or acoustic STM system.

However, it appears reasonable that not all visual stimulus materials are simply encoded acoustically or, for that matter, can be encoded acoustically in short-term storage. One recent investigation demonstrates that visually presented material can be encoded either acoustically or visually (Tversky, 1969). Tversky (1969) used same-different latency measures to sequentially presented stimulus pairs. She demonstrated that modality of encoding of the first stimulus can be experimentally manipulated. This was done by controlling the $S$ 's expectation of whether the second stimulus of a pair was a name or a face. Thus, the first stimulus can be

*This study is based on a thesis in partial fulfilment of the $B A$ degree with a major in psychology by the second author. Preparation of this report was supported by St. Francis Xavier University Council for Research Grant No. 131 to the first author.

$\$$ R equests for reprints should be sent to Ken den Heyer, Department of Psychology, St. Francis Xavier University, Antigonish, N.S., Canada. encoded verbally or visually, irrespective of whether or not it was a name or a face, depending on whether $S$ expected to match it with a name or face.

Posner, Boies, Eichelman, \& Taylor (1969) have demonstrated that orally presented material can be encoded visually. Posner \& Keele (1967) and Posner et al (1969) have demonstrated that the "same" times are faster when two letters are physically identical (e.g., AA) than when they have the same name (e.g., aA). The authors attributed this to the fact that in the physical match, Ss can match on the basis of a visual code, whereas in the identity match, an additional encoding procedure-naming-must take place. The interesting result of the report by Posner et al is that under appropriate instructions, if the first letter of a pair was presented orally and the second letter visually, the reaction time was as efficient as a physical match. This evidence strongly favors the hypothesis that auditory information can generate a visual code, which is then used in a matching process.

The above investigations clearly indicate the two-dimensional nature of short-term memory. An item may have either acoustic or structural (visual) representation in STM. The present investigation is concerned with stimulus materials which may be stored acoustically in part but which must have a structural representation in STM. Consider a 6 by 4 matrix containing eight randomly positioned letters. If $S$ is required to process both the position and identity of items, letters themselves may be encoded acoustically, but it is unlikely that the location of letters can be encoded in a similar fashion. It appears likely that location is mediated by some structural or visual code. Presumably, there is no reason why this visual code should not also contain identity information. This suggests that, in the task just described, identity information may suffer the advantage of dual representation in the form of both an acoustic and structural code in STM.

The present experiment is concerned with whether or not loss of information from a memory code requiring dual storage can occur in a selective manner. For instance, can position information be lost without an equal loss of identity information, or vice versa? To this end, different kinds of interpolating tasks-visual or verbal-will be used. The assumption here, of course, is that the nature of interpolated events is important in its effect on STM despite some evidence to the contrary (e.g., Howe, 1970).

\section{SUBJECTS}

The Ss were five female students at St. Francis Xavier University with normal, or corrected to normal, vision. APPARATUS

The materials were presented by a Scientific Prototype three-channel (Model GB) tachistoscope. Illumination for all fields was set at about $10 \mathrm{fL}$. In addition, use was made of a Bell and Howell solid-state cassette recorder, Model 294M.

STIMULUS MATERIALS

Three types of materials were used: stimulus cards, visual interpolated material, and verbal interpolated material. Each of the set of materials were designed as follows.

Stimulus Cards

Each card consisted of a 6 by 4 matrix containing eight randomly positioned letters with the following

Table 1

Mean Number of Letters and Positions Correct as a Function of the Three Types of Interpolated Activity

\begin{tabular}{llll}
\multicolumn{4}{c}{ of Interpolated A tivity } \\
\hline & \multicolumn{3}{c}{ Interpolated Activity } \\
\cline { 2 - 4 } Ss & None & Visual & Verbal \\
\hline & \multicolumn{3}{c}{ Position Information } \\
1 & $2.06^{*}$ & 0.00 & 0.79 \\
2 & 1.37 & 0.41 & 1.35 \\
3 & 3.63 & 0.27 & 1.72 \\
4 & 1.45 & 0.02 & 0.64 \\
5 & 1.66 & 0.21 & 0.75 \\
$\bar{X}$ & 2.03 & 0.18 & 1.08 \\
& & & \\
& & Identity Information \\
1 & 2.32 & 1.22 & 0.75 \\
2 & 1.08 & 0.02 & 0.00 \\
3 & 3.63 & 2.29 & 1.52 \\
4 & 2.65 & 0.87 & 1.33 \\
5 & 3.00 & 2.29 & 1.00 \\
\hline X & 2.54 & 1.33 & 0.92 \\
\hline
\end{tabular}

*Data is corrected for chance or guessing by the formula: $Y=\frac{24 x-64}{8+x}$ 
restraints: (1) summed over all cards, each letter occurred in each of the matrix positions once, (2) no letter was repeated on any particular card, (3) the letters $O$ and $U$ were not used (to avoid errors in scoring), and (4) no more than three letters were allowed to occupy any particular row or column on any one card. Seventy-two cards were needed, and these were constructed to satisfy the first three constraints. Letters used were Letraset Printpak No. 6. Matrix cells subtended a visual angle of $0.98 \times 0.74 \mathrm{deg}$ (see Fig. 1 for example card).

Visual Interpolated Material

Material consisted of 24 different cards, where each card contained three 2 by 4 matrices and each matrix contained three randomly positioned medium-sized dots transferred from Letraset No. 553. On each card, two of the 2 by 4 matrices had dots in identical positions, with the third matrix being different. The size of a matrix cell was approximately $0.74 \times 0.74 \mathrm{deg}$, and the separation between matrices was about $0.45 \mathrm{deg}$ (see Fig. 2 for example card).

Verbal Interpolated Material

This material consisted of a tape recording of 24 different series of five two-digit numbers. All numbers were multiples of five from 10 to 55 , inclusive. The order of the five numbers was random, and numbers were temporally spaced so that all five numbers were presented in a 10-sec period.

\section{PROCEDURE}

The procedure involved presentation of stimulus material for $50 \mathrm{msec}$ followed by one of three different 10 -sec periods of interpolated activity. The interpolated activity consisted of (1) visual discrimination, (2) verbal activity or addition, and (3) no activity. Ss were instructed to duplicate stimulus cards on appropriate response sheets $10 \mathrm{sec}$ after stimulus presentation.

Trials were blocked into groups of 12 for each of three conditions of interpolated activity. Ss received all 72 cards for each condition. The order of blocks of trials was determined randomly over three sessions. Ss were instructed as follows for each condition: No interpolated activity-" After presentation of

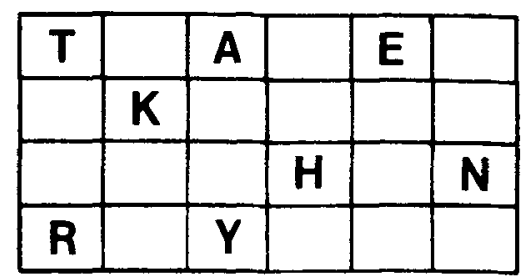

Fig. 1. An example stimulus card.
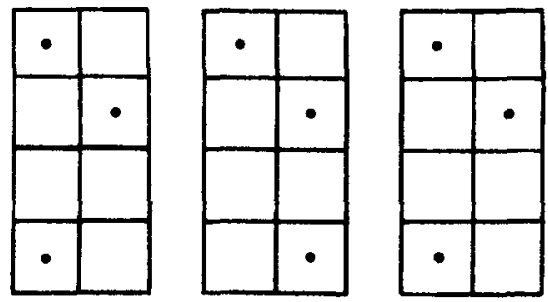

Fig. 2. An example of the material comprising the visual interpolated task.

stimulus a blank field will follow for $10 \mathrm{sec}$, and when this field terminates recall stimulus information." Visual interpolated activity-"After stimulus presentation a card with three distinct dot patterns will appear for $10 \mathrm{sec}$. Please indicate which one is different. When this card disappears then recall stimulus information." Verbal interpolated activity-"Please add five numbers. When the fifth number is presented give the total and report stimulus materials."

The Ss received one practice session before begining the three experimental sessions. Ss were instructed to fixate a fixation point before every trial. In responding, $S$ was required to place eight different letters in a response matrix. The response sheet was removed after every trial.

\section{RESULTS}

Scoring consisted of (1) correctly indicated positions, irrespective of content, and (2) correctly identified letters, irrespective of noted positions.
Means (72 trials) for the different measures were derived for each $\mathrm{S}$ and each interpolated condition. These means were corrected for chance or guessing (see Table 1).

A repeated-measure design (Subjects by Type of Interpolated Activity by Type of Information) was used to analyze the arcsine transformations of percentage loss data. The analysis of variance yielded a significant interaction effect $[F(1,4)=61.74$; $\mathrm{p}<.005]$, indicating the selective effects of the two interpolated tasks (see Fig. 1). Use of Sandler's A revealed that differences in performance loss between the two interpolated tasks, for each $S$, was larger for position information than for identity information $(A=.304$; $\mathrm{df}=4 ; \quad \mathrm{p}<.05)$. This last result indicates that the interpolated tasks had a more selective effect on the processing of position information than did the processing of identity information. The two main effects for either the type of interpolated task $[F(1,4)=6.91 ; p>.05]$ or type of information $[F(1,4)<1.0]$ did not prove to be significant.

The arcsine transformations of percentage loss data were subjected to one further analysis. Data were cast into a Ss by Type of Information by Direct-Indirect Interpolated Activity design. The analysis of variance yielded a significant direct-indirect effect $[F(1,4)=62.81 ; p<.005)$. The Type of Information by Direct-Indirect Interpolated Activity

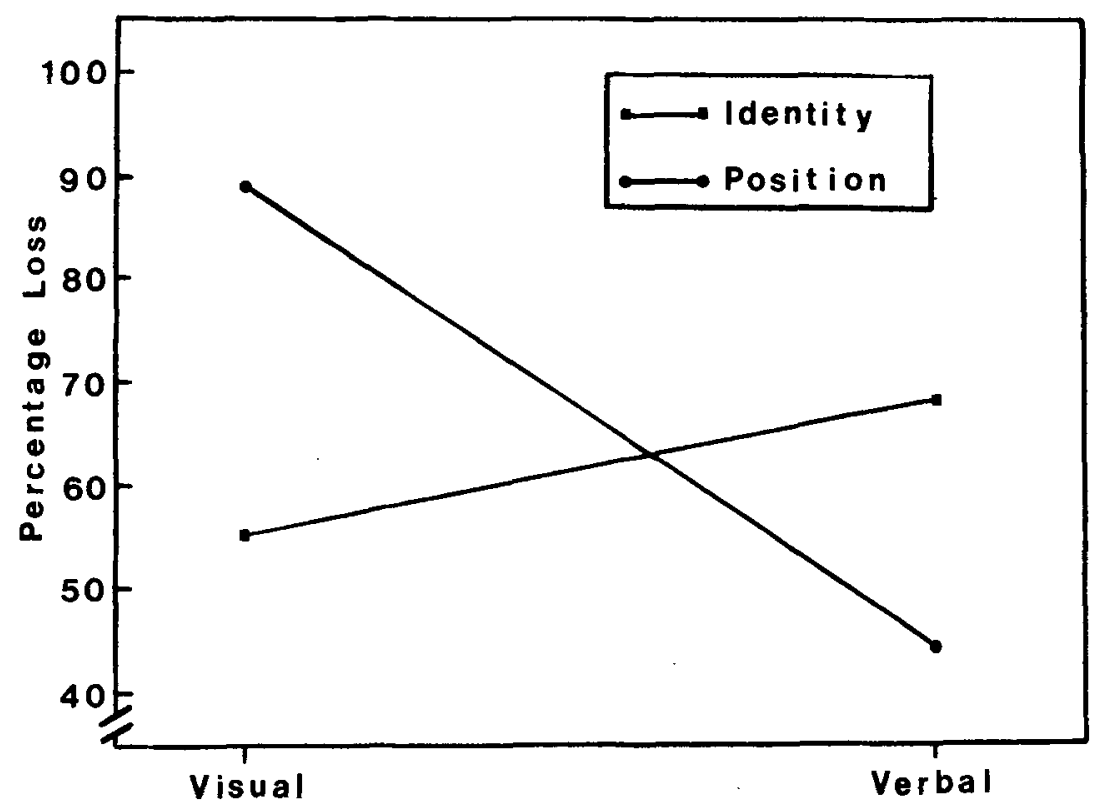

Type of Interpolated Task

Fig. 3. Percentage loss of the different kinds of information as a function of the type of interpolated task averaged over Ss. 
interaction effect did not prove to be significant $(F<1.0)$.

\section{DISCUSSION}

The large interaction effect demonstrates that information stored in STM is subject to selective loss as a function of the interpolated task between presentation and recall of stimulus materials. This rules out the possibility that loss of one kind of information (e.g., position information) will mean loss of the second type of information as well.

The interaction effect, however, can come about as the result of three different modes of information loss from STM, as has been pointed out by Cumming \& Coltheart (1969). One such mode would involve independence where loss of either type of information will not affect loss of the second type of information. The remaining two modes involve loss of one type of information which will not affect the loss of the second type of information without the reverse being true. Thus, it is possible that loss of position information will not affect loss of identity information, whereas loss of identity information would cause loss of position information. The third mode involves a reverse situation of the second mode. If either of the last two modes of information loss are operating, the results of the analysis of variance involving the direct-indirect interpolated activity factor should demonstrate an interaction effect. Since there was no hint of an interaction effect, it can be concluded that the information loss from STM occurs in a mutually independent manner. This is not to maintain that loss of one type of information has absolutely no effect on the storage of the other type of information, as is perhaps reflected in the loss of identity information as a function of the visual interpolated task and the loss of position information as a function of the verbal interpolated task. Other explanations, such as the fact that both interpolated tasks have visual and verbal components, are, of course, possible.

The fact that loss of information occurs selectively or independently to some extent indicates a two-dimensional representation of position and identity information in STM. This does not imply that these two dimensions of storage are acoustic and structural in nature, since such independence could exist within a particular modality. However, the fact that different STM modalities do exist is supported by the finding that differences in position performance loss as a function of the two interpolated tasks is larger than the similar difference for identity performance. This last finding can best be accounted for by a short-term memory code where position information is mediated by a structural code and identity information is mediated by the same structural code as well as an acoustic code. It should be noted that the last finding, and therefore the resulting interpretation, would not have been possible if the dual representation of identity was stored independently. Thus, loss of the acoustic component of a letter also implies loss of the structural component, and vice versa. REFERENCES

BADDELEY, A. D. Semantic and acoustic similarity in short-term memory. Nature. 1964, 204, 1116-1117.

CONRAD, R. Acoustic confusions in immediate memory. British Journal of Psychology, 1964, 55, 75-84.

CONRAD, R., \& HULL, A. J. Information. acoustic confusion, and memory span British Journal of Psychology, 1964, 55, 429-437.

CUMMING, G., \& COLTHEART, $M$. Short-term memory: Are item and position information stored independently? Psychonomic Science, 1969. 15, 216-218.

DALE, H. C, A., \& GREGORY, M. Evidence of semantic coding in short-term memory. Psychonomic Science, 1966, 5, 75-76.

HOWE, M. J. A. Introduction to human memory: A psychological approach. New York: Harper \& Row, 1970.

NEISSER, U. Cognitive psychology. New York: Appleton-Century-Crof ts, 1967.

POSNER, M. I., BOIES, S. J.. EICHELMAN, M. H., \& TAYLOR, R. L. Retention of visual codes and name codes of single letters. Journal of Experimental Psychology Monograph, 1969, 79(1, Part 2).

POSNER, M, I., \& KEELE, S, W. Decay of visual information from a single letter. Science, 1967, 158, 137-139.

SPERLING, G, A model for a visual memory task. Human Factors, 1963,5 19-31.

SPERLING, G. Successive approximations to a model for short-term memory. Acta Psychologica, 1967, 27, 285-292.

TVERSKY, B. Pictorial and verbal encoding in a short-term memory task. Pexception \& Psychophysics, 1969, 6, 225-233. 